\title{
A Novel Two-Stage Impulse Noise Removal Technique based on Neural Networks and Fuzzy Decision
}

\author{
V.Saradhadevi ${ }^{1}$ \\ Dr.V.Sundaram ${ }^{2}$ \\ ${ }^{1}$ Research Scholar, Karpagam University, Coimbatore, India. \\ ${ }^{2}$ Director of MCA, Karpagam Engineering College, Coimbatore, India.
}

\begin{abstract}
Image enhancement is plays a vital role in various applications. There are many techniques to remove the noise from the image and produce the clear visual of the image. Moreover, there are several filters and image smoothing techniques available in the literature. All these available techniques have certain limitations. Recently, neural networks are found to be a very efficient tool for image enhancement. A novel two-stage noise removal technique for image enhancement and noise removal is proposed in this paper. In noise removal stage, an adaptive two-level feed forward Neural Network (NN) with a Modified Levenberg-Marquardt training algorithm was used to eliminate the impulse noise. In the image enhancement stage, the fuzzy decision rules inspired by the Human Visual System (HVS) are used to categorize the image pixels into human perception sensitive class and nonsensitive class, and to enhance the quality of the image. The Hyper trapezoidal fuzzy membership function is used in the proposed technique. In order to improve the sensitive regions with higher visual quality, a Neural Network (NN) is proposed. The experiment is conducted with standard image. It is observed from the experimental result that the proposed two stage technique shows significant performance when compared to existing methods.
\end{abstract}

Keywords--- Fuzzy Decision, Impulse Noise, Peak Signal to Noise Ratio (PSNR)

\section{INTRODUCTION}

IMAGES are usually affected by means of impulse noise because of noisy sensors or channel transmission mistakes. The main aim of noise reduction is to smother the noise, and also probably to safeguard the sharpness of edge and feature information. The nonlinear filtering method-standard median (SM) [9], [10] filteraccording to order statistic, has been discussed to be usually better than linear filtering in reducing the impulse noise. Conversely, the median filter is inclined to blur fine details and demolish edges while smoothing out the impulse noise. For producing better result, the median filter has been altered in several manners. Those were expected to raise the signal preservation but relatively reduce the noise reduction capability. Applying these algorithms altogether.

In the majority of applications, it is very essential to suppress the impulse noise [11] from image because the performances of subsequent image processing techniques are strictly reliable on the accomplishment of image noise removal process [12]. Conversely, this is a very difficult in any image processing technique since the restoration filter must not alter the useful data in the image and conserve image data and texture during noise removal. The existing noise removal filters generally have the demerits of inducing unwanted distortions and blurring effects into the resulted image during noise removal phase.

So a novel approach is required for noise removal and image enhancement. Two-stage techniques that integrate noise identification and image enhancement have been proposed to eliminate the noise and keep the detail information well [13].

Since neural networks (NNs) have the ability to learn from examples, and fuzzy systems have the ability to deal with uncertainty, they also have a growing number of applications in image noise removal in the past few years [16]. Moreover, fuzzy techniques are also to detect impulse noise. These methods exhibit relatively better performance but require more computation and memory cost. It is desired to improve the quality of noise removal and reduce the time consumption at the same time.

A new two-stage noise removal technique to deal with impulse noise is proposed in this paper. An easily implemented NN is designed for fast and accurate noise detection such that various widespread densities of noisy pixels can be distinguished from the detail edge pixels well. After suppressing the impulse noise, the image quality enhancement is applied to compensate the corrupted pixels to enhance the visual quality of the resultant images. It consists of fuzzy decision rules based on the Human Visual System (HVS) for image analysis and an $\mathrm{NN}$ for image quality enhancement. If a noisecorrupted pixel is in the perception sensitive region, the proposed $\mathrm{NN}$ module is applied to this pixel for further quality compensation.

\section{RELATED WORKS}

Schulte et al., [1] proposed a fuzzy two-step filter for impulse noise reduction from color images. A novel method for suppressing impulse noise [4] from digital images is provided in this paper, in which a fuzzy detection process is followed by an iterative fuzzy filtering method [7]. The filter proposed by author is called as fuzzy two-step color filter. The fuzzy detection technique in this paper is generally based on the computation of fuzzy gradient values and on fuzzy reasoning. This step found out three different membership functions that are passed to the filtering phase. Those membership functions are used for fuzzy set impulse noise depiction. The proposed novel fuzzy technique is particularly developed for suppressing impulse noise from color images while preventing other image data and texture. Evaluation of the proposed noise filter indicates that it can be useful in removing the impulse noise efficiently from color images without affecting the valuable data in the image. 
Sun et al., [2] provided an impulse noise image filter using fuzzy sets. The successful use of fuzzy set theory performance on many domains, together with the increasing requirement for processing digital images, have been the main intentions following the efforts concentrated on fuzzy sets $[5,6]$. Fuzzy set hypothesis, contrasting with some other hypothesis, can offer us with knowledge-based and robust means for image processing. By calculating the fuzziness of the pixels affected degree and taking equivalent filter parameters, a novel image filter for suppressing the impulse noise is proposed in this paper. The proposed filter is more effective when compared to the median filter which is good for suppressing the impulse noise. Finally, the experimental result shows the feasibility of the proposed filtering technique.

Ibrahim et al., [3] given a simple adaptive median filter for the removal of impulse noise from highly corrupted images. This author proposed a simple, yet efficient technique to suppress impulse noise from noise affected images. This new technique composed of two phases. The first phase is to find the impulse noise affected pixels in the image. In this phase, depends on only the intensity values, the pixels are approximately separated into two classes, which are "noise-free pixel" and "noise pixel". Then, the second phase is to remove the impulse noise from the noise affected image. In this phase, only the "noise-pixels" are processed. The "noise-free pixels" are kept as such to the output image. This technique adaptively modifies the size of the median filter depends on the number of the "noise-free pixels" in the neighborhood. For the filtering process, only "noise-free pixels" are taken into account for the detection of the median value. The experiment evaluation for 100 test images indicates that the proposed technique outperforms some of the existing techniques, and can suppress the noise from highly corrupted images, up to noise concentration of $95 \%$. Average evaluation time required to completely process images of 1600 times 1200 pixels with $95 \%$ noise percentage is below 2.7 seconds. Because of its simplicity, this proposed technique is appropriate to be implemented in consumer electronics products such as digital television, or digital camera.
A novel impulse noise removal approach based on wavelet neural network is applied to restore digital images corrupted by impulse noise. Initially, wavelet neural network is applied to identify the noise-pixels and differentiate it from noise-free pixels. Then, the noise-pixels are categorized further by equivalent threshold and assigned the coefficient. Ultimately, the median filter is combined with the coefficient for the output. The proposed approach effectively eliminates the impulse noise while preserving more fine details. Visual evaluation and detailed statistical analysis show that the proposed technique is very significant than the conventional filters.

\section{SYSTEM ARCHITECTURE}

Optimal noise removal should delete the visible noise as cleanly as possible and maintain the detail information and natural appearance to obtain a natural-looking image.

In order to remove the impulse noise cleanly from input images without blurring the edge, the proposed system is divided into two stages.

\section{Impulse Noise Removal \\ 2. Image Enhancement}

In impulse noise removal stage, the impulse noise is removed without affecting too much detail information, and then, the image quality enhancement is applied to compensate the edge sharpness in the second stage. The two-level NN noise removal process is shown in Figure 1. Inside the first level, only the noisy pixels identified by the NN detection are processed with the $3 \times 3$ median filter. The second-level noise removal process is used to detect and remove the misclassified and the detected but un-removed noise pixels in the first-level noise removal process with an adaptive median filter.

The $3 \times 3$ window is used in this stage to get the features equivalent to the pixel $\mathrm{P}(\mathrm{O}, \mathrm{O})$ for noise detection. 


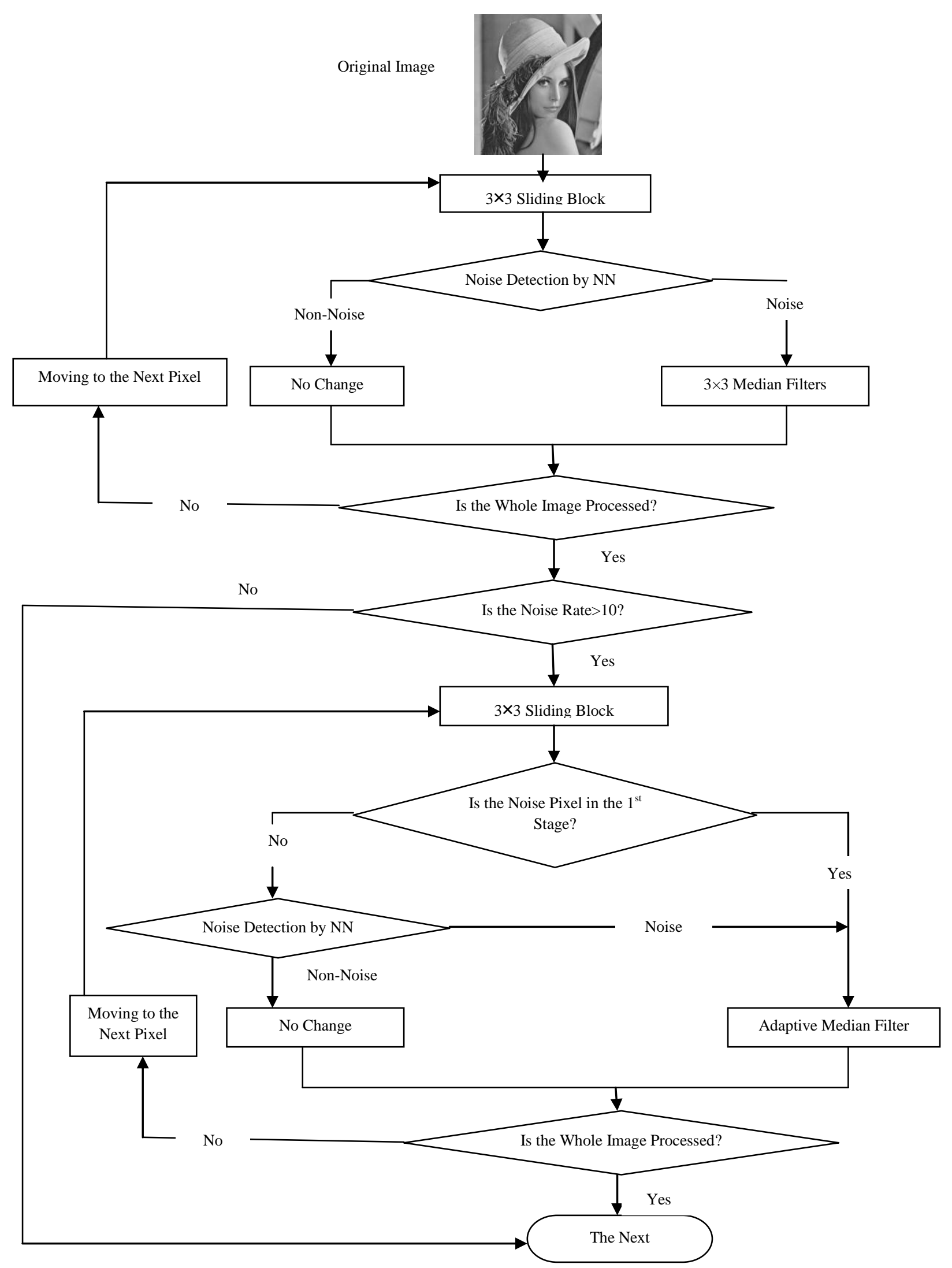

Figure 1: Procedure diagram of the two-level impulse noise removal 


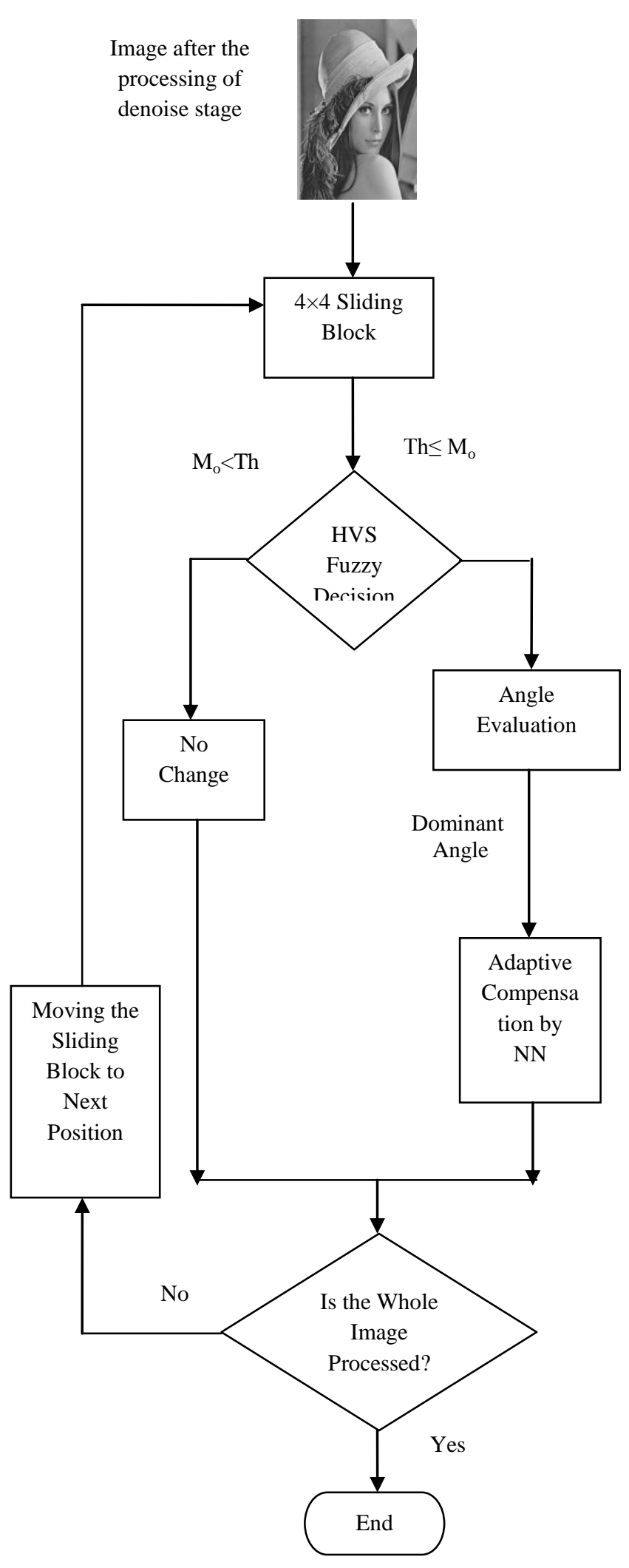

Figure 2: Procedure diagram of the image quality enhancement

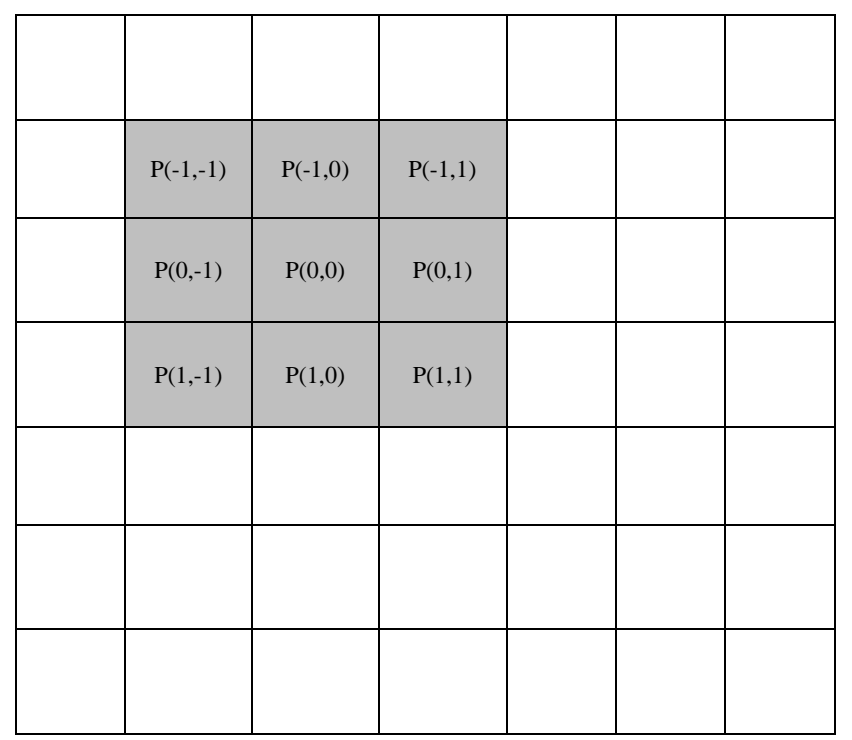

(a)

\begin{tabular}{|l|l|l|l|l|l|l|}
\hline & & & & & & \\
\hline & $\mathrm{O}(-1,-1)$ & $\mathrm{O}(-1,0)$ & $\mathrm{O}(-1,1)$ & $\mathrm{O}(-1,2)$ & & \\
\hline & $\mathrm{O}(0,-1)$ & $\mathrm{O}(0,0)$ & $\mathrm{O}(0,1)$ & $\mathrm{O}(0,2)$ & & \\
\hline & $\mathrm{O}(1,-1)$ & $\mathrm{O}(1,0)$ & $\mathrm{O}(1,1)$ & $\mathrm{O}(1,2)$ & & \\
\hline & $\mathrm{O}(2,1)$ & $\mathrm{O}(2,0)$ & $\mathrm{O}(2,1)$ & $\mathrm{O}(2,2)$ & & \\
\hline & & & & & & \\
\hline & & & & & & \\
\hline
\end{tabular}

(b)

Figure 3: Two sliding (overlapping) window blocks. (a) The $3 \times 3$ window applied to the first stage for impulse noise removal. (b) The $4 \times 4$ window applied to the second stage for image quality enhancement.

Figure 2 shows the schematic block diagram of the second stage image quality enhancement system. The proposed approach contains a fuzzy decision module, an angle evaluation module, and an adaptive compensation module. A fuzzy decision module based on the HVS categories each reference pixel $O(0,0)$ as sensible delineated edge or not. It is shown in figure 3. Depending on this category, the proposed adaptive NN compensation module is applied to the sensible delineated edge region. When the adaptive NN 
compensation is activated, the angle evaluation section will estimate the dominant orientation of the original image present in the sliding block as the input data of the proposed NN. The $4 \times 4$ window is applied at this stage to get the features equivalent to the pixel $O(0,0)$ for HVS-based image compensation.

The weighted compensation of $O(0,0)$ is applied to the noisecorrupted pixel $F(m, n)$ at the position $(m, n)$ in the sensible delineated edge region and can be presented as

$$
F(m, n)=\sum_{i=-1}^{2} \sum_{j=-1}^{2} O(i, j) W_{\theta}(i, j)
$$

where $W_{\theta}$ is derived from an $\mathrm{NN}$ after offline training. The $\mathrm{NN}$ is trained according to the edge angle of the reference image pixel to obtain the corresponding weights.

\section{PROPOSED IMPULSE NOISE REMOVAL}

\subsection{Impulse Noise Model}

Impulse noise is when the pixels are randomly failed and replaced by other values in an image. The image model containing impulse noise can be described as follows:

$$
X_{i j}=\left\{\begin{array}{c}
N_{i j}, \text { with probability } p \\
S_{i j}, \text { with probability } 1-p
\end{array}\right.
$$

where $S_{i j}$ represents the noiseless image pixel and $N_{i j}$ represents the noise substituting for the Original Pixel (OP).With the noise ratio $p$, only $p$ percent of the pixels in the image are replaced and others keep noise uncorrupted. In a variety of impulse noise models for images, fixed- and random-valued impulse noises are mostly discussed. Fixed-valued impulse noise, known as the "salt-and-pepper" noise, is made up of corrupted pixels whose values are replaced with values equal to the maximum or minimum (255 or 0 ) of the allowable range with equal probability $(p / 2)$. The random-valued impulse noise is made up of corrupted pixels whose values are replaced by random values uniformly distributed in the range within $[0,255]$. In this paper, both fixed and random-valued impulse noises are adopted as the noise model to test the system robustness.

\subsection{NN for Noise Detection}

As the residual noise greatly affects human perception, exact noise detection is an important factor for the noise removal.

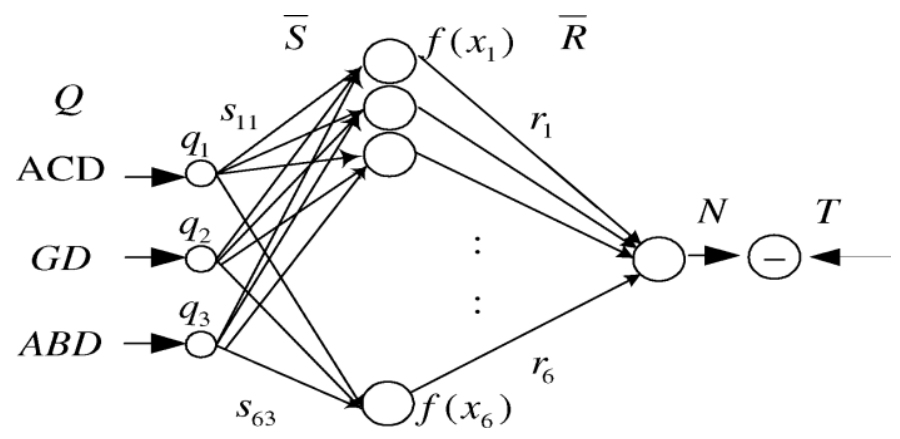

Figure 4: Proposed NN for Noise Detection

A NN with high accuracy and ability of dealing with various noisy images is proposed for noise detection. It is a 3-layer NN with one hidden layer, as shown in figure 4 . The input layer contains three nodes equivalent to the Gray-level Difference (GD), Average Background Difference (ABD), and Accumulation Complexity Difference $(A C D)$ in the $3 \times 3$ sliding window. The second layer is the hidden layer that contains six nodes, and the bipolar sigmoid function is applied as the activation function. The weighting vectors between the first and second layers, and between the second and third layers, are denoted as $S$ and $R$, respectively. The output layer contains one node that denotes the identified attribution of the pixel: "noise" or "non-noise," and moreover the bipolar sigmoid function is also used as the activation function. The three features in the input layer are discussed as follows.

1) Gray-Level Difference (GD): The GD represents the accumulated variations between the central pixel for identification and each surrounding local pixel. It is defined by

$$
G D=\sum_{i=-1}^{1} \sum_{\substack{j=-1 \\(i, j) \neq(0,0)}}^{1}|P(0,0)-P(i, j)|
$$

where $P(0,0)$ is the reference pixel and $P(i, j)$ is the surrounding local pixel.

The GD feature is considered to identify the noise over a flat area. It is expected that the corrupted pixels would yield much bigger differences as compared with the uncorrupted pixels.

2) Average Background Difference ( $A B D)$ : The surrounding pixels averaged as the Background Luminance (BL) of the sliding block which is then compared with the central pixel. This is an assistant feature to detect the noise. This feature, called the ABD, denoting the overall average variation with the central pixel in the block, is defined by

$$
A B D=\left|P(0,0)-\frac{\sum_{i=-1}^{1} \sum_{\substack{j=-1 \\(i, j) \neq(0,0)}}^{1} P(i, j)}{8}\right|
$$


The corrupted pixels provide bigger differences as compared with the clean ones. For the pixels in the texture area, the GD value is large but the ABD feature will be small.

3) Accumulation Complexity Difference (ACD): Accumulating the difference between each pixel in the $3 \times 3$ sliding block and its four neighboring pixels as defined next shows the structure information of the block

$$
\begin{aligned}
A C D=\sum_{i=-1}^{1} \sum_{j=-1}^{1} \mid 4 \times P(i, j)-P(i-1, j) \\
-P(i+1, j)-P(i, j-1) \\
-P(i, j+1) \mid
\end{aligned}
$$

In the edge area, the summation is lower than that in the noise-pixel area, though the GD difference might be similar. So, it provides an assistant feature between the edge and noise pixels.

\subsection{Modified Levenberg-Marquardt}

A Modified Levenberg-Marquardt algorithm is used for training the neural network.

Considering performance index is $F(w)=e^{T} e$ using the Newton method we have as:

$$
\begin{gathered}
W_{K+1}=W_{K}-A_{K}^{-1} \cdot g_{K} \\
A_{k}=\left.\nabla^{2} F(w)\right|_{w=w_{k}} \\
g_{k}=\left.\nabla F(w)\right|_{w=w_{k}} \\
{[\nabla F(w)]_{j}=\frac{\partial F(w)}{\partial w_{j}}=2 \sum_{i=1}^{N} e_{i}(w) \cdot \frac{\partial e_{i}(w)}{\partial w_{j}}}
\end{gathered}
$$

The gradient can write as:

$$
\nabla F(x)=2 J^{T} e(w)
$$

Where

$$
J(w)=\left[\begin{array}{cccc}
\frac{\partial e_{11}}{\partial w_{1}} & \frac{\partial e_{11}}{\partial w_{2}} & \ldots & \frac{\partial e_{11}}{\partial w_{N}} \\
\frac{\partial e_{21}}{\partial w_{1}} & \frac{\partial e_{21}}{\partial w_{2}} & \ldots & \frac{\partial e_{21}}{\partial w_{N}} \\
\cdot & \\
\frac{\partial e_{K P}}{\partial w_{1}} & \frac{\partial e_{K P}}{\partial w_{2}} & \ldots & \frac{\partial e_{K P}}{\partial w_{N}}
\end{array}\right]
$$

$J(w)$ is called the Jacobian matrix.

Next we want to find the Hessian matrix. The $k$, $j$ elements of the Hessian matrix yields as:

$$
\begin{gathered}
{\left[\nabla^{2} F(w)\right]_{k, j}=\frac{\partial^{2} F(w)}{\partial w_{k} \partial w_{j}}} \\
=2 \sum_{i=1}^{N}\left\{\frac{\partial e_{i}(w)}{\partial w_{k}} \frac{\partial e_{i}(w)}{\partial w_{j}}\right. \\
\left.+e_{i}(w) \cdot \frac{\partial^{2} e_{i}(w)}{\partial w_{k} \partial w_{j}}\right\}
\end{gathered}
$$

The Hessian matrix can then be expressed as follows:

$$
\nabla^{2} F(w)=2 J^{T}(W) \cdot J(W)+S(W)
$$

$$
S(w)=\sum_{i=1}^{N} e_{i}(w) \cdot \nabla^{2} e_{i}(w)
$$

If $\mathrm{S}(\mathrm{w})$ is small assumed, the Hessian matrix can be approximated as:

$$
\nabla^{2} F(w) \cong 2 J^{T}(w) J(w)
$$

Using (7) and (15) we obtain the Gauss-Newton method as:

$$
\begin{gathered}
W_{k+1}= \\
W_{k}-\left[2 J^{T}\left(w_{k}\right) \cdot J\left(w_{k}\right)\right]^{-1} 2 J^{T}\left(w_{k}\right) e\left(w_{k}\right) \\
\cong W_{k}-\left[J^{T}\left(w_{k}\right) \cdot J\left(w_{k}\right)\right]^{-1} J^{T}\left(w_{k}\right) e\left(w_{k}\right)
\end{gathered}
$$

The advantage of Gauss-Newton is that it does not require calculation of second derivatives.

There is a problem the Gauss-Newton method is the matrix $H=J^{T} J$ may not be invertible. This can be overcome by using the following modification.

Hessian matrix can be written as:

$$
G=H+\mu I
$$

Suppose that the eigenvalues and eigenvectors of $\mathrm{H}$ are $\left\{\lambda_{1}, \lambda_{2}, \ldots \ldots, \lambda_{n}\right\}$ and $\left\{z_{1}, z_{2}, \ldots \ldots, z_{n}\right\}$.Then:

$$
\begin{gathered}
G z_{i}=[H+\mu I] z_{i} \\
\quad=H z_{i}+\mu z_{i} \\
=\lambda_{i} z_{i}+\mu z_{i} \\
=\left(\lambda_{i}+\mu\right) z_{i}
\end{gathered}
$$


Therefore the eigenvectors of $\mathrm{G}$ are the same as the eigenvectors of $\mathrm{H}$, and the eigen values of $\mathrm{G}$ are $\left(\lambda_{i}+\mu\right)$. The matrix $\mathrm{G}$ is positive definite by increasing $\mu$ until $\left(\lambda_{i}+\mu\right)>0$ for all $i$ therefore the matrix will be invertible.

This leads to Levenberg-Marquardt algorithm:

$$
w_{k+1}=w_{k}-\left[J^{T}\left(w_{k}\right) J\left(w_{k}\right)+\mu I\right]^{-1} J^{T}\left(w_{k}\right) e\left(w_{k}\right)
$$

$$
\Delta w_{k}=\left[J^{T}\left(w_{k}\right) J\left(w_{k}\right)+\mu I\right]^{-1} J^{T}\left(w_{k}\right) e\left(w_{k}\right)
$$

As known, learning parameter, $\mu$ is illustrator of steps of actual output movement to desired output. In the standard LM method, $\mu$ is a constant number. This paper modifies LM method using $\mu$ as:

$$
\mu=0.01 e^{T} e
$$

Where $e$ is a $k \times 1$ matrix therefore $e^{T} e$ is a $1 \times 1$ therefore $\left[J^{T} J+\mu I\right]$ is invertible.

Therefore, if actual output is far than desired output or similarly, errors are large so, it converges to desired output with large steps.

Likewise, when measurement of error is small then, actual output approaches to desired output with soft steps. Therefore error oscillation reduces greatly.

\subsection{Noise Removal Algorithm}

After the first level, the image noise density is calculated to decide whether the second level is necessary or not by the precise detection procedure. By the experiments, it is observed that when the noise density is below $10 \%$, only a one-level noise removal process is enough. More residual noises will occur when the noise density increases. In this case, the second-level noise removal process is essential to detect and remove the residual noises.

As the local features may influence the correctness of the detection part and the median filter may still retain certain noises, the residual noise pixels are detected and removed with an adaptive median filter in the second level. If there are more than $30 \%$ noisy pixels in this image, it is identified as a highly corrupted region and the $5 \times 5$ median filter is applied for processing. Otherwise, the $3 \times 3$ median filter is used to process the noisy pixel. The proposed adaptive twolevel noise removal technique is very efficient to suppress the impulse noise as well as to preserve the sharpness of edges and detail information.

\section{PROPOSED IMAGE QUALITY ENHANCEMENT}

The conventional median filtering techniques have the limitation of blurring details and cause artifacts around edges. In order to compensate the edge sharpness, image quality enhancement is applied to the modified pixels. As the first stage has eliminated the visible noise, the second stage focuses the image enhancement on the edge region. For image analysis, the properties of the HVS are used to acquire the features of images. Thus, region which would worth quality enhancement is realized, since human eyes would be usually more sensitive to this region. For sensitive regions, an adaptive NN is used to enhance the visual quality to match the characteristics of human visual perception.

\subsection{HVS-Directed Image Analysis}

A novel fuzzy decision system motivated by the HVS is proposed to categorize the image into human perception sensitive and nonsensitive regions. There are three input variables: Visibility Degree (VD); Structural Degree (SD); and Complexity Degree (CD), and one Output Variable (Mo) in the proposed fuzzy decision system.

Visibility Degree (VD): The capability of human eyes to identify the magnitude difference between an object and its background depends on the BL. Figure 5 shows the actual visibility thresholds called JND corresponding to different BLs, and they were verified by a subjective experiment [15]. The experiments were conducted in a dark room and a square area was located in the center of a flat field of constant gray level. Through varying the amplitude of the object, the visibility threshold for each gray level was determined when the object was just noticeable. 


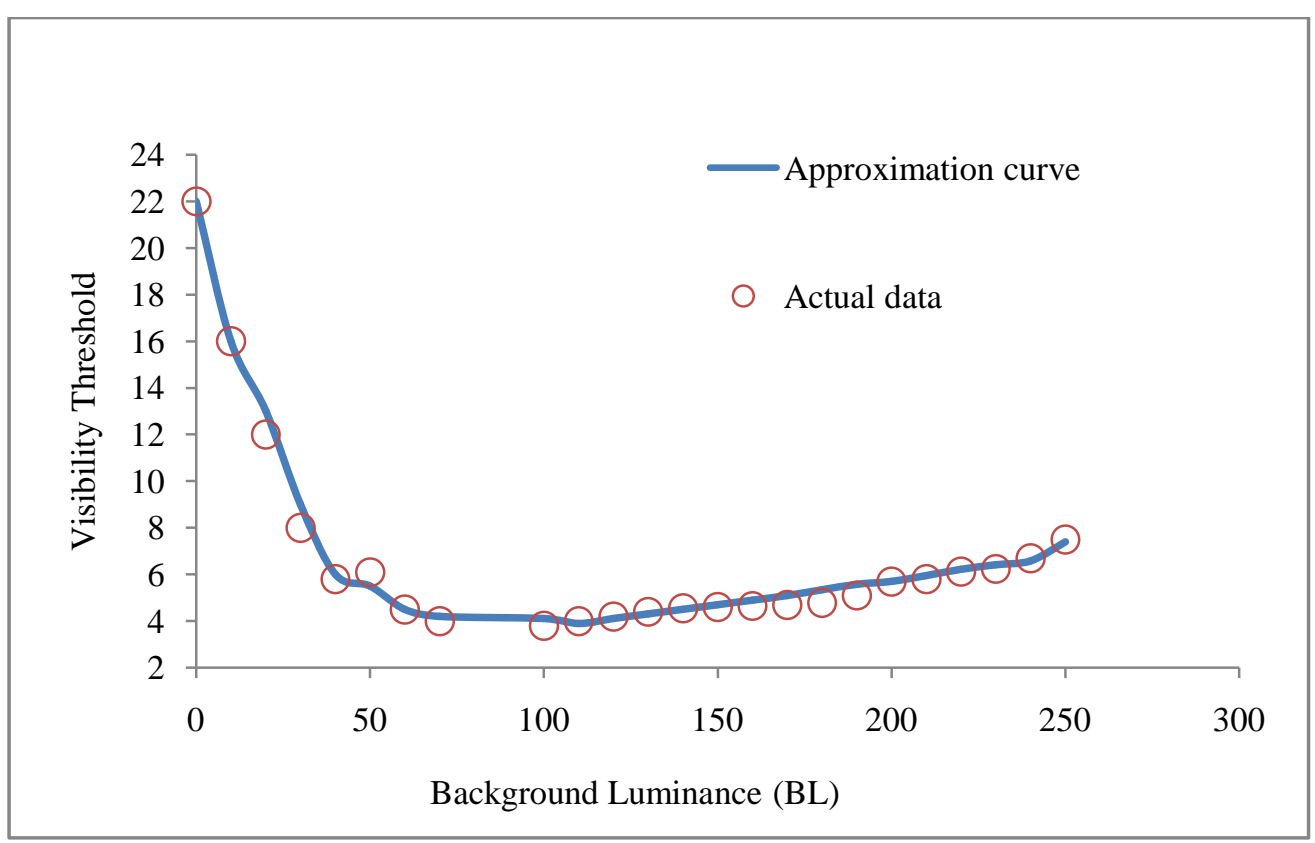

Figure 5: Visibility thresholds corresponding to different BLs

It is observed from figure 5 that the visibility threshold is lower when the $\mathrm{BL}$ is within the interval from 70 to 150 , and the visibility threshold will increase if the BL becomes darker or brighter away from this interval. In addition, a high visibility threshold will occur when the $\mathrm{BL}$ is in a very dark region.

$\mathrm{BL}$ is the average luminance of the sliding block proposed to approximate the actual BL and can be calculated by

$$
\begin{gathered}
B L=\frac{1}{23} \sum_{i=-1}^{2} \sum_{j=-1}^{2} O(i, j) \times B(i, j) \\
B(i, j)=\left[\begin{array}{llll}
2 & 2 & 2 & 1 \\
2 & 0 & 2 & 1 \\
2 & 2 & 2 & 1 \\
1 & 1 & 1 & 1
\end{array}\right]
\end{gathered}
$$

and the denominator 23 in (22) is the weighted sum of all elements in (23) for normalization. The weighting coefficients of B decrease as the corresponding distance away from the reference pixel increases to estimate the average BL. Feature D is the difference between the maximum and minimum pixel values in the sliding block and can be calculated by

$$
D=\max (o(i, j))-\min (o(i, j))
$$

A nonlinear function $\mathrm{V}$ (BL) is also designed to approximate the relation between the visibility threshold and BL (as figure. 5), and can be represented as

$$
V(B L)=20.66 e^{-0.03 B L}+e^{0.008 B L}
$$

The parameter of 20.66 is obtained by substituting 0 for BL in the nonlinear approximation equation by setting the coefficient of e 0.008 BL to be 1 .

The first input variable of the fuzzy decision system, VD, is defined as the difference between D and V (BL) and can be represented as

$$
V D=D-V(B L)
$$

If $\mathrm{VD}>0$, it means the magnitude difference between the object and its background exceeds the visibility threshold and the object is sensible. Otherwise, this object is not sensible.

The other two input variables, $\mathrm{SD}$ and $\mathrm{CD}$, are used to indicate whether the pixels in the sliding block own the edge structure.

Structural Degree (SD): SD shows if the sliding block is a high contrast region, and the pixels in the block can be evidently partitioned into two clusters. It is calculated by

$=\frac{|\max (O(i, j))-\operatorname{mean}(O(i, j))-[\operatorname{mean}(O(i, j))-\min (o(i, j))]|}{\max (O(i, j))-\min (O(i, j))}$

Where

$$
\operatorname{mean}(O(i, j))=\frac{1}{16} \sum_{i=-1}^{2} \sum_{j=-1}^{2} O(i, j)
$$




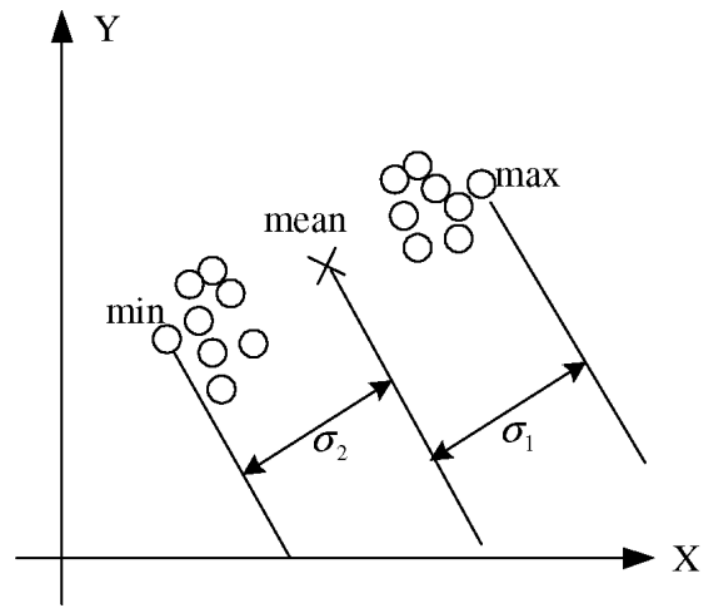

(a)

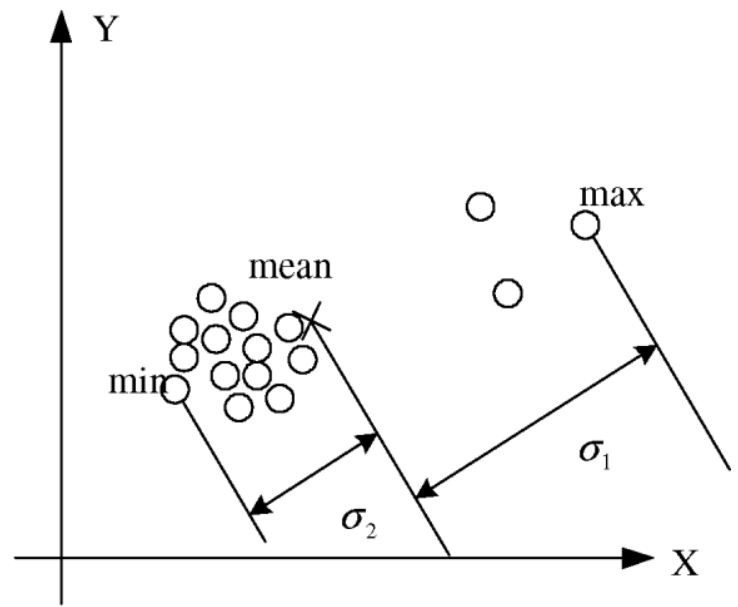

(b)

Figure 6: Illustration of the relation between the SD parameter and the distribution of pixels in a sliding block

According to figure 6, equation (11) can be expressed as $\quad \mid \sigma_{1}-$ $\sigma_{2} /\left(\sigma_{1}+\sigma_{2}\right)$. So, the SD has been normalized to $[0,1]$ and this rule can also be applied to images with a different intensity range. If SD is small and $\sigma_{2}$ and $\sigma_{1}$ are close, it means the pixels in the block can be partitioned into two even clusters. The block may contain edge or texture structure. On the contrary, if SD is a large value, $0<\left|\sigma_{1}-\sigma_{2}\right|$,

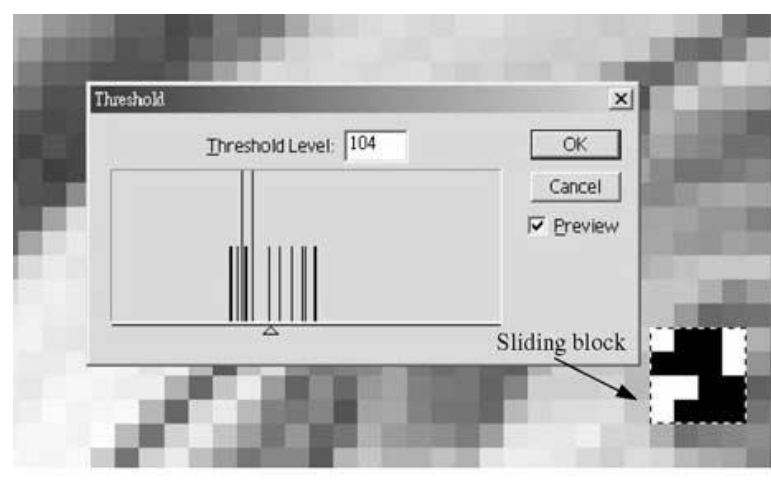

(a) it means the pixel number of one cluster and that of the other cluster are not even; thus, the block may contain noise.

Complexity Degree (CD): Figure 7(a) and (b) shows a texture structure and a delineated edge structure in a sliding block, respectively.

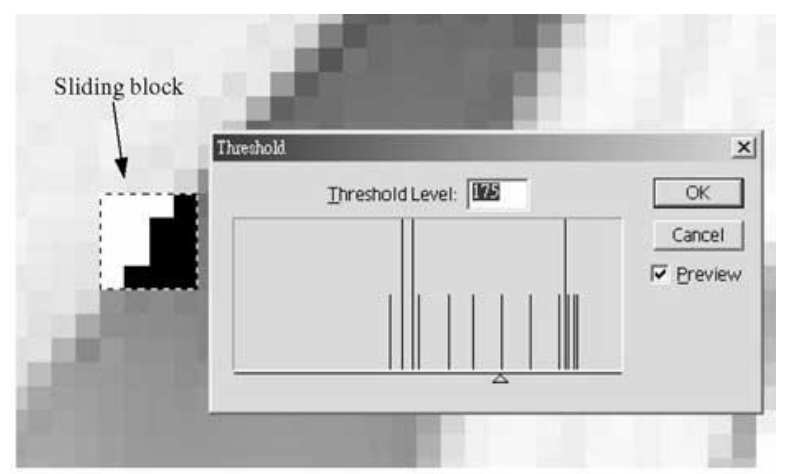

(b)

Figure 7: Portions of (a) the sliding block including texture structure, and (b) the sliding block including edge structure.

In these two plots, pixel numbers of the two clusters are the same. Hence, the SD values equivalent to these two structures are close. As the proposed $\mathrm{NN}$ is used to compensate the sensitive regions, such as figure 7(b), a CD input variable based on the differential process is used to tell the delineated edge structure from the texture structure. It is calculated by

$$
\begin{aligned}
C D=\sum_{i=-1}^{2} \sum_{j=-1}^{2} \mid 4 O^{\prime}(i, j) & \\
& -\left[O^{\prime}(i+1, j)+O^{\prime}(i-1, j)\right. \\
& \left.+O^{\prime}(i, j-1)+O^{\prime}(i, j+1)\right] \mid
\end{aligned}
$$

Where $O^{\prime}(i, j)$ is the binarized version of $O(i, j)$. Assuming mean $(\mathrm{O})$ is the mean gray value of the sliding block, $O^{\prime}(i, j)$ is defined as

$$
O^{\prime}(i, j)=\left\{\begin{array}{c}
1, \text { if } O(i, j) \geq \operatorname{mean}(O) \\
0, \text { otherwise }
\end{array}\right.
$$

In (29), each pixel in the $4 \times 4$ sliding block takes the 4 - directional local gradient operation and the $\mathrm{CD}$ is the summation of the 16 local gradient values. If the $\mathrm{CD}$ is a large value, it means the block may contain texture structure. On the contrary, if the $\mathrm{CD}$ is a small value, the block may contain delineated edge structure. 


\subsubsection{Hyper Trapezoidal Fuzzy Membership Function}

The proposed system uses the Hyper Trapezoidal fuzzy Membership function.

Hyper trapezoidal membership functions are defined by prototype points and a crispness factor. In a fuzzy partitioning of an $\mathrm{N}$ dimensional space, let each fuzzy set, $S_{i}$ be defined by a prototype point, $\lambda_{i}$. Furthermore, let the partitioning of the space also be parameterized by a crispness factor, $\sigma$. The prototype point, $\lambda_{i}$ has a degree of membership in set, $S_{i}$, of $\mu_{i}\left(\lambda_{i}\right)=1$ and a degree of membership in set $S_{j}$, of $\mu_{j}\left(\lambda_{j}\right)=1$ where $j \neq i$.

The crispness factor, $0 \leq \sigma \leq 1$, determines how much ambiguity exists between the sets of the partitioning. For $\sigma=1$, no fuzziness exists between the sets and the partitioning is equivalent to a minimum distance classifier. For fuzzy sets $\sigma<1$. One way to define the crispness factor is using Figure 8 and equation (31).

$$
\sigma=\frac{2 \alpha}{d}
$$

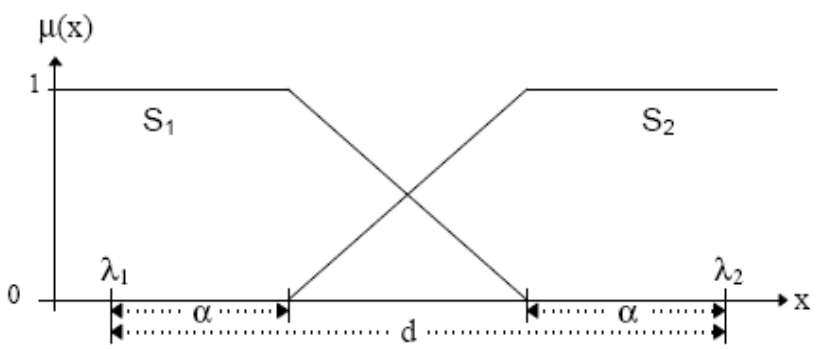

Figure 8: Defining Crispness of a partitioning

The crispness factor establishes fuzzy space between the prototype points. The prototype points are selected as ideal representatives of each fuzzy set. Then, the designer's selection of $\sigma$ specifies the ratio of $\alpha$ and d.

The next step in the derivation is the definition of a suitable distance measure relating the distance from the crisp input to two prototype points. This distance measure is a ratio of the distance between two prototype points, and the difference in the distances from the crisp input to the two prototype points. For fuzzy sets $S_{i}$ and $S_{j}$, with prototype points $\lambda_{i}$ and $\lambda_{j}$, and a crisp input $\Lambda$, that distance measure is

$$
\rho_{i \mid j}(\Lambda)=\frac{\left|\vec{v}_{i}\right|^{2}-\left|\vec{v}_{j}\right|^{2}}{\left|\vec{v}_{j i}\right|^{2}}
$$

Where $\vec{v}_{j i}$ is a vector from $\lambda_{i}$ to $\lambda_{j} ; \vec{v}_{i}$ is a vector from $\lambda_{i}$ to $\Lambda$. This distance measure is used to determine if the crisp input $\Lambda$ lies completely in fuzzy set $i$, or completely in fuzzy set $j$, or in the fuzzy region between the two sets.

The third step in the derivation of hyper trapezoidal membership functions is determining the degree of membership that $\mathrm{L}$ has in set $\mathrm{i}$, given that set $\mathrm{j}$ is the only other set in the partition. Suppose fuzzy sets $\mathrm{i}$ and $\mathrm{j}$ are the only two sets defined in an $\mathrm{N}$-dimensional space. Using the distance measure of equation (32), that degree of membership is

$$
\begin{aligned}
& \mu_{i \mid j}(\Lambda) \\
& =\left\{\begin{array}{cc}
0 ; & \rho_{i \mid j}(\Lambda) \geq 1-\sigma \\
1 ; & \rho_{i \mid j}(\Lambda) \leq \sigma-1 \\
\frac{\vec{v}_{j i} \cdot \vec{v}_{j}-\frac{\sigma}{2}\left|\vec{v}_{j i}\right|^{2}}{(1-\sigma)\left|\vec{v}_{j i}\right|^{2}} & \text { otherwise }
\end{array}\right\}
\end{aligned}
$$

For the first case in equation (33), $\Lambda$ lies completely in fuzzy set $\mathrm{j}$. For the second case, $\Lambda$ lies completely in fuzzy set $i$. The third case is the case of $\Lambda$ being in the transition from set $i$ to set $j$.

The numerical value of Mo after defuzzification is compared with a threshold value, Th, where Th is preferably set as the value 5 by experiments. When $\mathrm{Mo} \geq \mathrm{Th}$, the adaptive NN compensation module with angle evaluation would be chosen; otherwise, the OP value would be used.

\subsection{Angle Evaluation}

As Mo $\geq T h$, the fuzzy system identifies the reference pixel as sensible delineated edge and the trained adaptive NN model is chosen for quality enhancement according to its corresponding edge angle. The angle evaluation is performed to determine the dominant orientation of the sliding block. When the orientation angle of $\mathrm{O}(\mathrm{i}, \mathrm{j})$ denoted as A ( $i, j)$ is computed, the luminance values of the OPs nearby $\mathrm{O}(\mathrm{i}, \mathrm{j})$ are used for the following computations:

$$
\begin{aligned}
D x(i, j)=O(i- & 1, j-1)+2 O(i-1, j)+O(i \\
& -1, j+1)-(O(i+1, j-1) \\
& +2 O(i, j+1)+O(i+1, j+1)) \\
D y(i, j)=O(i- & 1, j-1)+2 O(i, j-1)+O(i+1, j \\
& -1)-(O(i-1, j+1) \\
& +2 O(i, j+1)+O(i+1, j+1)) \\
& \\
A(i, j)= & -\frac{180}{\pi}\left[\tan ^{-1}\left(\frac{D y(i, j)}{D x(i, j)}\right)\right]
\end{aligned}
$$

Where $-1 \leq i \leq 2$ and $-1 \leq j \leq 2$.

The obtained angle of each pixel in the sliding window is quantized into eight quantization sectors such as $\theta=22.5 \times \mathrm{k}$ (in degrees), where $\mathrm{k}=0,1, \ldots, 7$. Assuming $\theta$ is the quantized angle for most pixels in the window; it is regarded as the dominant orientation of the reference edge pixel. The corresponding weighting coefficient $\mathrm{W}_{\theta}$ derived from the offline training $\mathrm{NN}$ is adopted for compensation filtering. 


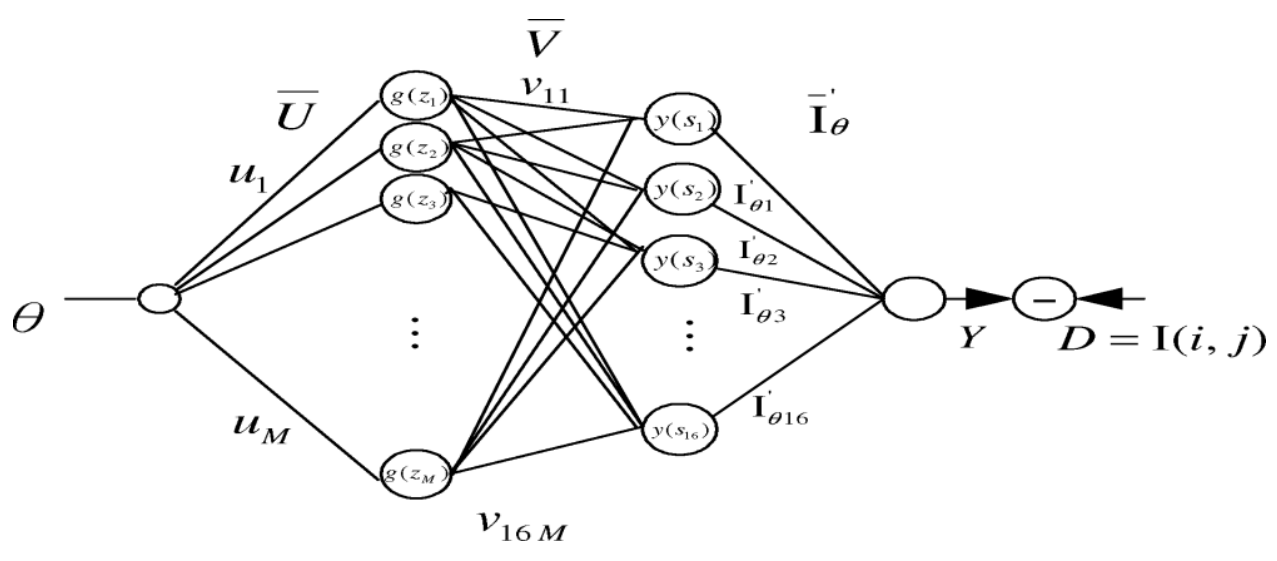

Figure 9: Proposed feed forward NN for image quality enhancement

\subsection{NN-Based Image Compensation}

The function of the proposed $\mathrm{NN}$ is to obtain the weights $\mathrm{W} \theta$ defined in (1), where $\theta$ represents the quantized dominant orientation of the reference pixel. Thus, the proposed $\mathrm{NN}$ is used to obtain eight sets of weighting matrices through training.

Each weighting matrix $\mathrm{W}_{\theta}$ can be represented as

$$
W_{\theta}(i, j)=\left[\begin{array}{llll}
w_{-1-1} & w_{-10} & w_{-11} & w_{-12} \\
w_{0-1} & w_{00} & w_{01} & w_{02} \\
w_{1-1} & w_{10} & w_{11} & w_{12} \\
w_{2-1} & w_{20} & w_{21} & w_{22}
\end{array}\right]
$$

In order to use supervised learning algorithms to train the proposed $\mathrm{NN}$, several clean image portions with dominant orientation are used as training patterns. Assuming a clean image portion is denoted as I, the noise-corrupted version of I has been processed by the proposed noise removal method in the first stage and the filtered result is denoted as $I^{\prime}$. According to figure 9 , let $I^{\prime}(i, j)$ be the reference pixel, where $\mathrm{O}(0,0)=I^{\prime}(i, j)$, and it is classified as an edge pixel with dominant orientation $\theta$ after angle evaluation. The input of the NN can be defined as IP $=\theta$ and the network output is the compensated pixel value of $I^{\prime}(i, j)$. The pixel value of $I(i, j)$ obtained from the clean original image is used as the desired output of the $\mathrm{NN}$ for training.

\section{EXPERIMENTAL RESULTS}

For experimenting the proposed filtering technique, several $512 \mathrm{X}$ 512 grayscale images affected by the noise with noise occurrence of $1 \%$ to $50 \%$ is considered. The result of the proposed filter is compared with several existing filters such as median filter and two stage filter with different window size. The quantitative measures used for comparison is the Peak Signal-to-Noise Ratio (PSNR) between the original and restored images and average execution time. PSNR value is evaluated by using the following equation:

$$
P S N R=10 \log _{10}\left(\frac{\sum_{i=0}^{N-1} \sum_{j=0}^{N-1} 255^{2}}{\sum_{i=0}^{N-1} \sum_{j=0}^{N-1}[I(i, j)-Y(i, j)]^{2}}\right)
$$

Where $\{I(i, j)\}$ and $\{Y(i, j)\}$ are the original and restored images, respectively.

Table 1 provides the comparison of the proposed filter with median filter ( $3 \mathrm{X} 3$ window size), median filter ( $5 \mathrm{X} 5$ window size) and two stage filter. The experimentation is performed at different noise level such as $1 \%, 5 \%, 10 \%, 20 \%, 40 \%$ and $50 \%$.

From the table 1, it can be observed that the PSNR value resulted in $5 \%$ noise affected image is 29.6 for the median filter of window size $3 \times 3,30.2$ for the median filter of window size $5 \times 5,34.7$ for the two stage filter whereas it is higher for the proposed Two Stage filter with hyper trapezoidal fuzzy membership function and Modified LM i.e., 38.2 .

When the image is affected by higher noise i.e., 50\%, other filters results only less PSNR value i.e., 13.6 for the median filter of window size $3 \times 3,14.3$ for the median filter of window size $5 \times 5$, 28.4 for the two stage filter whereas it is higher for the proposed Two Stage filter with hyper trapezoidal fuzzy membership function and Modified LM i.e., 33.9. When the overall PSNR is considered, the proposed filter shows better PSNR values when compared to the conventional filters.

Table 1: Comparative results in PSNR of different filtering methods for various percentages of noise (Lena image)

\begin{tabular}{|c|c|c|c|c|c|c|}
\hline Filter & $1 \%$ & $5 \%$ & $10 \%$ & $20 \%$ & $40 \%$ & $50 \%$ \\
\hline $\begin{array}{c}\text { Median Filter } \\
(3 X 3)\end{array}$ & 31.3 & 29.6 & 25.9 & 23.2 & 20.9 & 13.6 \\
\hline $\begin{array}{c}\text { Median Filter } \\
\text { (5X5) }\end{array}$ & 32.5 & 30.2 & 27.1 & 25.1 & 21.3 & 14.3 \\
\hline $\begin{array}{c}\text { Two Stage } \\
\text { filter }\end{array}$ & 36.8 & 34.7 & 33.2 & 32.4 & 31.2 & 28.5 \\
\hline $\begin{array}{c}\text { Two Stage } \\
\text { filter with } \\
\text { hyper } \\
\text { trapezoidal } \\
\text { fuzzy } \\
\text { membership } \\
\text { function and } \\
\text { Modified LM }\end{array}$ & 39.7 & 38.2 & 37.6 & 35.2 & 34.8 & 33.9 \\
\hline
\end{tabular}


Table 2 shows the execution time taken for the proposed filter and the different existing image filters with the noise rate as $80 \%$. From the table, it can be observed that execution time required for the median filter with 3 X 3 window size is 14 seconds, median filter with 5 X 5 window size is 16 seconds, two stage filter is 13 seconds, whereas, the execution time required by the proposed technique is 9 seconds. This clearly indicates that the overall execution time required by the proposed filter is lesser when compared to the existing filters.

Table 2: Comparative results in average execution time of different filtering methods for Lena image corrupted image by $80 \%$ salt and pepper noise

\begin{tabular}{|c|c|c|c|c|}
\hline Filters & $\begin{array}{c}\text { Median } \\
\text { Filter } \\
(3 \times 3)\end{array}$ & $\begin{array}{c}\text { Median } \\
\text { Filter } \\
(5 \times 5)\end{array}$ & $\begin{array}{c}\text { Two Stage } \\
\text { filter with } \\
\text { LM } \\
\text { training } \\
\text { Algorithm }\end{array}$ & $\begin{array}{c}\text { Two Stage } \\
\text { filter with } \\
\text { hyper } \\
\text { trapezoidal } \\
\text { fuzzy } \\
\text { membership } \\
\text { function and } \\
\text { Modified } \\
\text { LM }\end{array}$ \\
\hline Time & 14 & 16 & 13 & 9 \\
\hline (seconds) & 14 & 16 & & \\
\hline
\end{tabular}

\section{CONCLUSION}

A novel two-stage noise removal technique is proposed in this paper. In the first stage, a two level noise removal procedure with NN-based noise detection was applied to remove the eliminate noise. In the second stage, a fuzzy decision rule inspired by the HVS was proposed to categorize pixels of the image into human perception sensitive and nonsensitive classes. An NN is proposed to enhance the sensitive regions to perform better visual quality. The neural network is trained using the Modified Levenberg-Marquardt algorithm. Moreover, the hyper trapezoidal member function is used which provides significance performance. The proposed technique is experimented with 512 X 512 grayscale image with the noise occurrence of $1 \%$ to $50 \%$. The PSNR value obtained for the proposed technique is higher when compared to the existing filtering techniques. Moreover, the execution time taken by the proposed approach is very significant when compared with the existing approaches. Thus the proposed technique is very efficient when compared with the conventional methods in perceptual image quality, and it can provide a quite a stable performance over a wide variety of images with various noise densities.

\section{REFERENCES}

[1] Schulte, S., De Witte, V., Nachtegael, M., Van der Weken, D. and Kerre, E.E., "Fuzzy Two-Step Filter for Impulse Noise Reduction From Color Images", IEEE Transactions on Image Processing, Vol. 15, No. 11, Pp. 3567 - 3578, 2006

[2] Sun Zhong-gui, Chen Jie and Meng Guang-wu, "An Impulse Noise Image Filter Using Fuzzy Sets", International Symposiums on Information Processing (ISIP), Pp. 183 - 186, 2008 .
[3] Ibrahim, H., Kong, N.S.P. and Theam Foo Ng, "Simple adaptive median filter for the removal of impulse noise from highly corrupted images", IEEE Transactions on Consumer Electronics, Vol. 54, No. 4, Pp. 1920 - 1927, 2008.

[4] Abreu, E., Lightstone, M., Mitra, S.K. and Arakawa, K., "A New Efficient Approach for the Removal of Impulse Noise from Highly Corrupted Images", IEEE Transaction on Image Processing, Vol. 5, No. 6, Pp. 1012-1025, 1996.

[5] Russo, F. and Ramponi, G., "A Fuzzy Filter for Images Corrupted by Impulse Noise", IEEE Signal Processing Letters, Vol. 3, No. 6, Pp. 168-170, 1996.

[6] Choi, Y.S. and Krishnapuram, R., "A Robust Approach to Image Enhancement Based on Fuzzy Logic", IEEE Transaction on Image Processing, Vol. 6, No. 6, Pp. 808-825, 1997.

[7] Boskovitz, V. and Guterman, H., "An Adaptive Neuro-Fuzzy System for Automatic Image Segmentation and Edge Detection", IEEE Transactions on Fuzzy Systems, Vol. 10, No. 2, Pp. 247-262, 2002.

[8] Chao Deng; Ji Yu An; “An Impulse Noise Removal Based on a Wavelet Neural Network", Second International Conference on Information and Computing Science, ICIC '09, Volume 2, pages 71-74, 2009.

[9] J. B. Bednar and T. L. Watt, "Alpha-trimmed means and their relationship to median filters," IEEE Trans. Acoust., Speech, Signal Process., vol. ASSP-32, no. 1, pp. 145-153, Feb. 1984.

[10] D. A. F. Florencio and R.W. Schafer, "Decision-based median filter using local signal statistics," in Proc. SPIE Symp. Vis. Commun. Image Process., 1994, vol. 2308, pp. 268-275.

[11] S. J. Ko and Y. H. Lee, "Center weighted median filters and their applications to image enhancement," IEEE Trans. Circuits Syst., vol. 38, no. 9, pp. 984-993, Sep. 1991.

[12] T. Chen and H. R. Wu, "Impulse noise removal by multi-state median filtering," in Proc. Int. Conf. Acoust., Speech, Signal Process., vol. 4, pp. 2183-2186, Jun. 2000.

[13] X. Li and M. Orchard, "True edge-preserving filtering for impulse noise removal," in presented at the 34th Asilomar Conf. Signals, Syst. Comput., Pacific Grove CA, Oct. 2000.

[14] D. Zhang and Z.Wang, "Impulse noise detection and removal using fuzzy techniques," Electron. Lett., vol. 33, no. 5, pp. 378379, Feb. 1997.

[15] C. H. Chou and Y. C. Li, "A perceptually tuned subband image coder based on the measure of just-noticeable-distortion profile," IEEE Trans. Fuzzy Syst., vol. 3, no. 3, pp. 467-476, Dec. 1995.

[16] P. Civicioglu, "Using uncorrupted neighborhoods of the pixels for impulsive noise suppression with ANFIS," IEEE Trans. Image Process., vol. 16, no. 3, pp. 759-773, Mar. 2007. 